\title{
Project Background and Methodological Aspects
}

The general aim of the European Nutrition and Health Report 2009 was to provide a comprehensive up-to-date documentation on the nutrition and health situation in the Europe Union.

The specific objectives were

- To describe trends in food supply

- To compare average daily individual food availability

- To evaluate food consumption in adults based on representative nutrition surveys

- To evaluate energy and nutrient intake in all age groups based on national/ representative nutrition surveys

- To inform about diet-related health indicators and status

- To analyze food and nutrition policies in European countries.

The methods implied collecting and critically reviewing available data on the most common indicators used for the assessment of the nutrition and health situation of 25 European countries. The main task of the participating partners was the collection of all relevant national data and in consequence the provision of clarifications in describing and analyzing the available data in a comparable manner.

The implementation strategy was a simple, feasible four-step process (fig. 3.1):

Step 1: Selection and definition of nutrition and health indicators to be included in the report

To decide on the nutrition and health indicators to be considered following steps were taken:

- Based on the experience gained from the European Nutrition and Health Report 2004 [Elmadfa et al., 2005] a template with questions concerning the fields of information, survey, methods of analysis, references and cut-off points and others was prepared by the coordinating center and sent out to the participating countries prior to the project's kick-off meeting in October 2007.

- During the kick-off meeting a representative of each country presented available data from the respective country. 


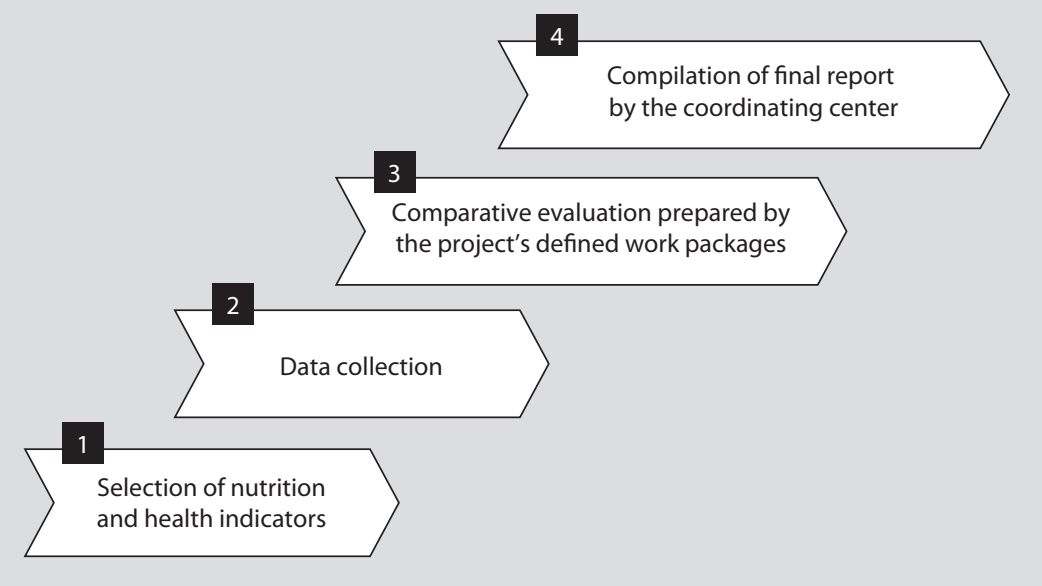

Fig. 3.1. Implementation strategy of the project Report on Nutrition and Health Status.

- After the kick-off meeting the template was revised. A more specific and accurate questionnaire with questions on available data for specified age groups was developed and sent again to the partners. All 25 countries answered the questionnaire, on that background it was possible to decide which health indicators to include in the European Nutrition and Health Report 2009.

- Recommendations and the experience of former European projects such as EFCOSUM [EFCOSUM, 2002], ECHI [ECHI, 2005] and the European Nutrition and Health Report 2004 [Elmadfa et al., 2005] were taken into account.

Step 2: Collection of existing published and authorized unpublished data in each participating country

To facilitate and harmonize data collection on nutrition and health indicators templates were developed and sent to the participating organizations.

In addition, data from already existing databases such as the WHO Health for All database [WHO, 2009a], the EFSA concise food consumption database [EFSA, 2008a] and the Food Balance sheets of the FAO [FAO, 2009] were collected.

Data on food and nutrition policy were collected by a separate questionnaire developed by the project's specific Work Package, Data on food availability at the household level were derived from the DAFNE databank (the task of another project's work package).

Step 3: Comparative evaluation of available data

Data were described and analyzed by the work packages with assistance of project partners. During the final plenary meeting of the project in June 2009 the results were discussed with the consortium.

Step 4: Compilation of the final report by the coordinating center

The main report consists of the chapters 'Trends of Average Food Supply in the European Union', 'Food Availability at the Household Level in the European Union', 


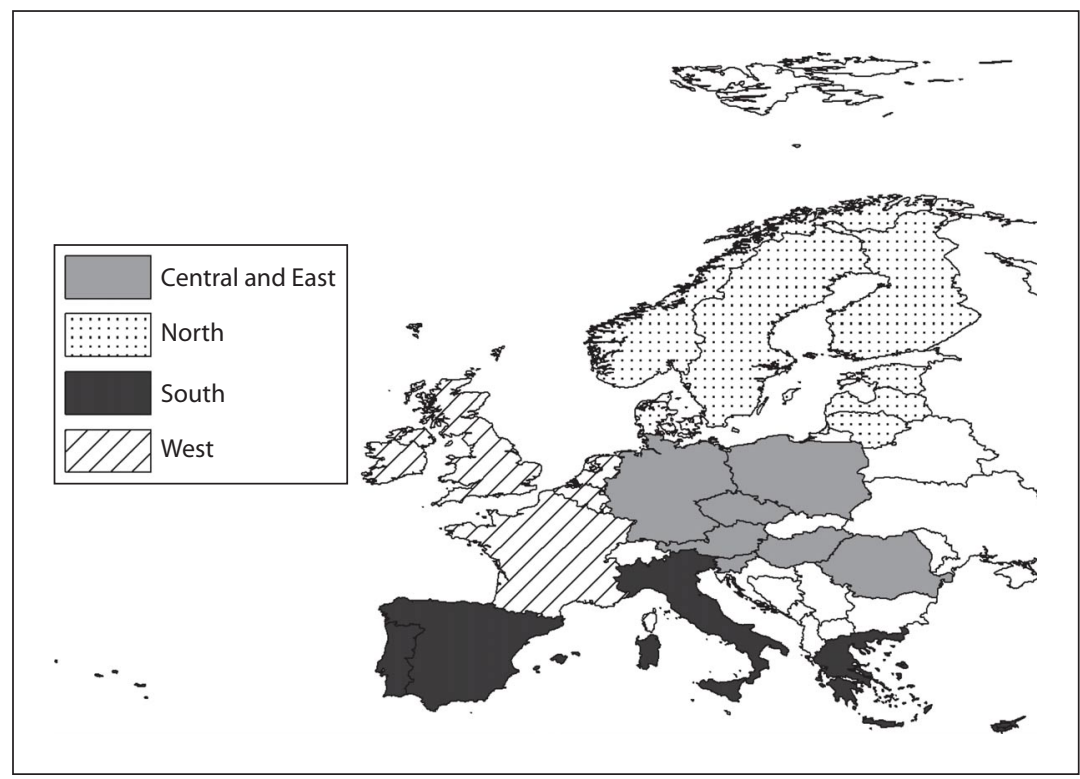

Fig. 3.2. Regions and countries of the European Nutrition and Health Report 2009.

'Food Consumption in Adults on the Basis of Dietary Surveys in European Countries', 'Energy and Nutrient Intake in the European Union', 'Health and Lifestyle Indicators in the European Union, 'Food and Nutrition Policies in European Countries' and a 'General Discussion'. Additional information on the nutrition and health situation in the participating countries is given in the chapter national reports (pp. 250-405).

In order to identify present region-specific data on diet, nutrition, physical activity, and diet-related health indicators of the European Union population following regions were defined and data analyzed accordingly (fig. 3.2):

- North: Denmark, Estonia, Finland, Latvia, Lithuania, Norway, Sweden

- South: Cyprus, Greece, Italy, Portugal, Spain

- Central and East: Austria, Czech Republic, Germany, Hungary, Poland, Romania, Slovenia

- West: UK, Belgium, France, Ireland, The Netherlands, Luxembourg, United Kingdom During the project it was not possible to collect raw data. Not all countries could provide data on nutrition and health indicators according to predefined age groups and to calculate prevalence of overweight and obesity according to the suggested methods. Nevertheless, also those data were included to get the most comprehensive picture. However, data have to be interpreted cautiously taking into consideration that not exactly the same methods were used to obtain the data.

In order to properly interpret data derived from different methods in chapters 7 and 8.1 , no means were calculated but minimum and maximum values were given as a range. 